Correction

\title{
Correction: An Extended Damage Plasticity Model for Shotcrete: Formulation and Comparison with Other Shotcrete Models
}

\section{Matthias Neuner * (D), Peter Gamnitzer and Günter Hofstetter}

Unit for Strength of Materials and Structural Analysis, Institute of Basic Sciences in Engineering Science, Innsbruck University, Technikerstr. 13, A-6020 Innsbruck, Austria; Peter.Gamnitzer@uibk.ac.at (P.G.);

Guenter.Hofstetter@uibk.ac.at (G.H.)

* Correspondence: Matthias.Neuner@uibk.ac.at; Tel.: +43-512-507-61521

Academic Editor: Erik Schlangen

Received: 4 January 2018; Accepted: 12 January 2018; Published: 15 January 2018

The authors would like to correct following typing errors: For (3) and (4), the correct expressions are given as

$$
\begin{aligned}
f_{\mathrm{p}}\left(\bar{\sigma}_{\mathrm{m}}, \bar{\rho}, \theta, q_{\mathrm{h}}\left(\alpha_{\mathrm{p}}\right), t\right)= & \left(\left(1-q_{\mathrm{h}}\left(\alpha_{\mathrm{p}}\right)\right)\left(\frac{\bar{\rho}}{\sqrt{6} f_{\mathrm{cu}}(t)}+\frac{\bar{\sigma}_{\mathrm{m}}}{f_{\mathrm{cu}}(t)}\right)^{2}+\sqrt{\frac{3}{2}} \frac{\bar{\rho}}{f_{\mathrm{cu}}(t)}\right)^{2} \\
& +m_{0} q_{\mathrm{h}}^{2}\left(\alpha_{\mathrm{p}}\right)\left(\frac{\bar{\rho}}{\sqrt{6} f_{\mathrm{cu}}(t)} r(\theta)+\frac{\bar{\sigma}_{\mathrm{m}}}{f_{\mathrm{cu}}(t)}\right)-q_{\mathrm{h}}^{2}\left(\alpha_{\mathrm{p}}\right), \\
g_{\mathrm{p}}\left(\bar{\sigma}_{\mathrm{m}}, \bar{\rho}, q_{\mathrm{h}}\left(\alpha_{\mathrm{p}}\right), t\right)= & \left(\left(1-q_{\mathrm{h}}\left(\alpha_{\mathrm{p}}\right)\right)\left(\frac{\bar{\rho}}{\sqrt{6} f_{\mathrm{cu}}(t)}+\frac{\bar{\sigma}_{\mathrm{m}}}{f_{\mathrm{cu}}(t)}\right)^{2}+\sqrt{\left.\frac{3}{2} \frac{\bar{\rho}}{f_{\mathrm{cu}}(t)}\right)^{2}}\right. \\
& +q_{\mathrm{h}}^{2}\left(\alpha_{\mathrm{p}}\right)\left(\frac{m_{0} \bar{\rho}}{\sqrt{6} f_{\mathrm{cu}}(t)}+\frac{m_{\mathrm{g}}\left(\bar{\sigma}_{\mathrm{m}}\right)}{f_{\mathrm{cu}}(t)}\right),
\end{aligned}
$$

corresponding to the respective time-independent equations in [1].

For (14), parameter $c_{\mathrm{f}}$ is computed as

$$
c_{\mathrm{f}}=\left.\frac{\mathrm{d} \beta_{\mathrm{f}}^{\mathrm{II}}}{\mathrm{d} t}\right|_{t=t_{\mathrm{f}}}-2 d_{\mathrm{f}} t_{\mathrm{f}} .
$$

The authors would like to express their gratitude to Anna-Lena Hammer from Ruhr University Bochum, Germany for finding this error.

The changes do not affect the results. The manuscript will be updated, and the original one will remain available on the article webpage.

\section{Reference}

1. Grassl, P.; Jirásek, M. Damage-plastic model for concrete failure. Int. J. Solids Struct. 2006, 43, 7166-7196.

(c) 2018 by the authors. Licensee MDPI, Basel, Switzerland. This article is an open access article distributed under the terms and conditions of the Creative Commons Attribution (CC BY) license (http://creativecommons.org/licenses/by/4.0/). 